\title{
Development and improvement of Bragg coherent diffraction imaging for expanding observable particle-size range
}

\author{
Norihiro Oshime $^{1 *}$, Kenji Ohwada ${ }^{1}$, Kento Sugawara ${ }^{1}$, Tetsuro Ueno ${ }^{1}$, Akihiko Machida $^{1}$, Tetsu Watanuki ${ }^{1}$, \\ Kenji Ishii ${ }^{1}$, Hidenori Toyokawa ${ }^{1,2}$, and Yoshihiro Kuroiwa ${ }^{3}$ \\ ${ }^{1}$ Synchrotron Radiation Research Center, Kansai Photon Science Institute, Quantum Beam Science Research Directorate, National \\ Institutes for Quantum and Radiological Science and Technology (QST), SPring-8, 1-1-1 Kouto, Sayo, Hyogo 679-5148, Japan, \\ ${ }^{2}$ Japan Synchrotron Radiation Research Institute, Kouto 1-1-1, Sayo-cho, Sayo-gun, Hyogo 679-5198, Japan, 3Graduate School of \\ Advanced Science and Engineering, Hiroshima University, Higashihiroshima, Hiroshima, 739-8526, Japan \\ oshime.norihiro@qst.go.jp
}

Functional properties such as dielectric constant [1] and hydrogen storage [2] in fine crystalline materials often exhibit particle size effects. Understanding the phenomena with size effects and utilizing their functions require observing the structure in conjunction with shape, size, and heterogeneity information.

We report the development and improvement of an apparatus for Bragg coherent x-ray diffraction imaging (Bragg-CDI) [3] at BL22XU in SPring- 8 . The achieved observable particle size was $40-500 \mathrm{~nm}$ and $\mathrm{Pd}(\sim 40 \mathrm{~nm})$ and ferroelectric barium titanate $\left(\mathrm{BaTiO}_{3}, \mathrm{BTO}, 200 \sim 500 \mathrm{~nm}\right)$ fine crystals were investigated.

This study aims to achieve two primary goals. (1) The first is to reduce background noise due to x-ray scattering by air. To this end, we newly prepared a vacuum chamber for samples, enabling us to obtain high-contrast x-ray diffraction pattern for a shorter time. (2) The second is to optimize a real-space constraint; our modified phase-retrieval algorithm can use appropriate real-space constraints with shrinking [4] support to refine the phase distribution.

We succeeded in expanding the observable particle-size range from 100-300 [3] to 40-500 $\mathrm{nm}$ [5] for the Bragg-CDI at BL22XU in SPring-8. The reconstructed three-dimensional image showed the outer shape, size, and internal phase (strain) for a single particle. A single 500-nm BTO particle showed a straight and sharp antiphase-boundary shape, whereas smaller BTO particles showed different phase boundary shapes. The present Bragg-CDI thus allows the observation of the outer shape, size, and inner phase distribution for a single particle with a size of tens to hundreds of nanometres, which may lead to a simple understanding of mesoscale ferroelectricity.

This work was partly supported by JSPS Grant-in-Aid for Scientific Research (Grant Nos. JP19H02618, JP18H03850, JP18H05518, JP19H05819, JP19H05625) and The Murata Science Foundation.

Full author list: N. Oshime, K. Ohwada, K. Sugawara, T. Abe, R. Yamauchi, T. Ueno, A. Machida, T. Watanuki, S. Ueno, I. Fujii, S. Wada, R. Sato, T. Teranishi, M. Yamauchi, K. Ishii, H. Toyokawa, K. Momma and Y. Kuroiwa.

[1] T. Hoshina, J. Ceram. Soc. Jpn. 121, 156 (2013).

[2] M. Yamauchi, R. Ikeda, H. Kitagawa, and M. Takata, J Phys C, 112, 3294 (2008).

[3] K. Ohwada, K. Sugawara, T. Abe, T. Ueno, A. Machida, T. Watanuki, S. Ueno, I. Fujii, S. Wada, and Y. Kuroiwa, Jpn. J. Appl. Phys. 58, SLLA05 (2019).

[4] S. Marchesini, H. He, H. Chapman, S. Hau-Riege, A. Noy, M. Howells, U. Weierstall, and J. Spence, Phys. Rev. B 68, 140101 (2003).

[5] N. Oshime, K. Ohwada, K. Sugawara, T. Abe, R. Yamauchi, T. Ueno, A. Machida, T. Watanuki, S. Ueno, I. Fujii, S. Wada, R. Sato, T. Teranishi, M. Yamauchi, K. Ishii, H. Toyokawa, K. Momma and Y. Kuroiwa, Jpn. J. Appl. Phys. (in press). https://doi.org/10.35848/1347-4065/ac148b

\section{Keywords: Bragg coherent diffraction imaging; coherent diffraction imaging}

Any acknowledgements authors wish to make should be included at the end of the manuscript with no heading (use Acknowledgement style, Times New Roman 10 pt, italics). 